Pacific Journal of Mathematics

MESOCOMPACTNESS AND RELATED PROPERTIES 


\section{MESOCOMPACTNESS AND RELATED PROPERTIES}

\section{J. Mancuso}

This paper is concerned with some of those generalizations of paracompactness which can arise by broadening the concept of local finiteness, e.g., metacompactness, in contrast to those which come about by varying the power of an open cover, e.g., countable paracompactness. Quite recently, several generalizations of the first type have been studied. These include mesocompactness and sequential mesocompactness, strong and weak cover compactness, and Property $Q$.

In $\S 1$, the notion of metacompactness (=pointwise paracompactness) is used to establish a hierarchy among these concepts, and in regular $r$-spaces, some of these notions are shown to be equivalent to paracompactness. In $\S 2$, it is shown that mesocompactness is an invariant, in both directions, of perfect maps and that unlike paracompact spaces, there exists a mesocompact $T_{3}$ space which is not normal, and a mesocompact $T_{2}$ space which is not regular.

Throughout this paper, a space will mean a Hausdorff space. A convergent sequence in a space will mean the sequence and its limit, and we will use $\mathrm{C} 1(A)$ to denote the closure of $A$.

1. Related properties. We will begin with some pertinent definitions.

Definition 1.1. A family $\mathscr{F}$ of sets in a space $X$ is called compact-finite (convergent sequence finite) if every compact set (convergent sequence) in $X$ meets at most finitely many members of $\mathscr{F}$. A space $X$ is called mesocompact (sequentially mesocompact) if every open cover of $X$ has a compact-finite (convergent sequence finite) open refinement (see [3]). We will use the abbreviation cs-finite for convergent sequence finite.

Definition 1.2. A cover $\mathscr{V}^{-}$of a space $X$ is called strong cover compact if whenever $\left\{V_{i} ; i \in N\right\}$ is a countably infinite subcollection of distinct elements of $\mathscr{Y} ; p_{i}$ and $q_{i} \in V_{i}$ for each $i$, with $p_{i} \neq p_{j}$ and $q_{i} \neq q_{j}$ for $i \neq j$ and the point set $\left\{p_{i} ; i \in N\right\}$ has a limit point in $X$, then the point set $\left\{q_{i} ; i \in N\right\}$ has a limit point in $X$.

DEFINITION 1.3. A space $X$ is strong cover compact if every open cover of $X$ has a strong cover compact open refinement. We will use the abbreviation scc for strong cover compact. 
If "countably" is replaced by "uncountably" in Definition 1.2, we obtain the notion of a weak cover compact cover. Then if every open cover of $X$ has a weak cover compact open refinement, we say that $X$ is weak cover compact, denoted by wcc (see [4]).

Definition 1.4. A cover $\mathscr{Y}$ of a space $X$ is said to have Property $Q$ if whenever $\left\{V_{i} ; i \in N\right\}$ is a countably infinite subcollection of distinct elements of $\mathscr{Y}$; with $p_{i}$ and $q_{i} \in V_{i}$ for each $i$ and $\left\{p_{i} ; i \in N\right\}$ converges to $p$ in $X$, then $\left\{q_{i} ; i \in N\right\}$ converges to $p$ in $X$.

Definition 1.5. A space $X$ has Property $Q$ if every open cover of $X$ has an open refinement with Property $Q$ (see [16]).

REMARK 1.6. It is immediate that paracompact implies mesocompact implies sequentially mesocompact implies metacompact. Moreover, since a locally finite cover vacuously satisfies scc, wcc and Property $Q$, a paracompact space is sce, wec and has Property $Q$.

We will often refer to a minimality property of point-finite covers [5, p. 160]: If $\mathscr{A}$ is a point-finite open cover of a space $X$, then there exists an irreducible sub-cover, i.e., a sub-cover that, when any single set is removed, is no longer a cover of $X$. This property was especially utilized in [4].

Proposition 1.7. A space $X$ has Property $Q$ if and only if $X$ is sequentially mesocompact.

Proof. Since a $c s$-finite cover vacuously satisfies Property $Q$, we need only establish the necessity. Let $\mathscr{C}$ be an open cover of $X$ and let $\mathscr{Y}$ be an open refinement with Property $Q$. Now some subcollection $\mathscr{Y}^{\prime}$ of $\mathscr{Y}$ is point-finite and covers $X$ [4, Th. 1]. Let $\mathscr{V}^{\prime \prime}$ be an irreducible sub-cover of $\mathscr{y}^{\prime \prime}$. We claim $\mathscr{y}^{\prime \prime}$ is cs-finite. For suppose $\left\{p_{i} ; i \in N\right\} \cup\{p\}$ is a convergent sequence in $X$ which meets infinitely many members of $\mathscr{V}^{\prime \prime}$, say $\left\{V_{i} ; i \in N\right\}$. Since $\mathscr{Y}^{\prime \prime}$ is pointfinite, there exist subsequences $\left\{p_{i_{n}} ; n \in N\right\}$ of $\left\{p_{i} ; i \in N\right\}$ and $\left\{V_{i_{n}} ; i \in N\right\}$ of $\left\{V_{i} ; i \in N\right\}$ such that $p_{i_{n}} \in V_{i_{n}}$ for each $n$. If we let $V_{p}$ be a member of $\mathscr{Y}^{\prime \prime}$ containing $p$, we can use the minimality of $\mathscr{V}^{\prime \prime}$ to pick a sequence $\left\{q_{i_{n}} ; n \in N\right\}$ such that $q_{i_{n}} \in V_{i_{n}}-V_{p}$ for each $n$. Clearly, $\left\{q_{i_{n}} ; n \in N\right\}$ does not converge to $p$ and yet $\left\{p_{i_{n}} ; n \in N\right\}$ does. This is a contradiction and the proof is complete.

CoROllary 1.8. A developable space is metrizable if and only if it has Property $Q$. 
Proof. In a developable space, metrizability is equivalent to sequential mesocompactness [3, Th. 4.2], which is equivalent to Property $Q$ by Proposition 1.7.

REMARK 1.9. Corollary 1.8 is obtained in [4, Corollary 5] by different means.

Both parts of the following proposition have similar proofs, each using the essential features of the proof of Theorem 8 or Theorem 13 in [4].

Notation. If $\mathscr{\mathscr { C }}$ is a collection of sets, $\mathscr{K}^{*}=U\{h ; h \in \mathscr{H}\}$.

Proposition 1.10. (a) If $X$ is regular wec and metacompact, $X$ is mesocompact. (b) If $X$ is scc and metacompact, $X$ is mesocompact.

Proof. To prove (a), we may assume $X$ has an open cover $\mathscr{G}$ which has no countable sub-cover, or else $X$ is Lindelof and hence paracompact. We can obtain an open refinement $\mathscr{Y}$ of $\mathscr{G}$ which is wcc, point-finite and irreducible. We claim $\mathscr{V}^{-}$is compact-finite. Suppose there exists a compact subset $K$ of $X$ which meets infinitely many members of $\mathscr{V}^{-}$say $\left\{V_{i} ; i \in N\right\}$. Then pick a distinct sequence $\left\{p_{i} ; i \in N\right)$ such that $p_{i} \in K \cap V_{i}$ for each $i$. Since $K$ is compact, the point set $\left\{p_{i} ; i \in N\right\}$ has a limit point in $X$.

Let $\left\{V_{\beta} ; \beta \in B\right\}$ be an uncountable subcollection of $V-\left\{V_{i} ; i \in N\right\}$, which exists since $\mathscr{V}$ refines $\mathscr{G}$. Since $\mathscr{V}$ is minimal, for each $i \epsilon$ $N$ and $\beta \in B$, let $q_{i} \in V^{*}-V_{i}$ and $q_{\beta} \in \mathscr{Y}^{*}-V_{\beta}$. Then the subcollection $\left\{V_{i} ; i \in N\right\} \cup\left\{V_{\beta} ; \beta \in B\right\}$ and the point sets $\left\{p_{i} ; i \in N\right\} \cup\left\{q_{\beta} ; \beta \in B\right\}$ and $\left\{q_{i} ; i \in N\right\} \cup\left\{q_{\beta} ; \beta \in B\right\}$ contradict the wec property of $\mathscr{Y}$; and so $\mathscr{Y}$ is compact-finite.

To prove (b), we let $\mathscr{U}$ be an open cover of $X$. Without the regularity of $X$, Theorem 8 in [4] can still be used to obtain an open refinement $\mathscr{Y}$ of $\mathscr{U}$ which is scc, point-finite and irreducible. A slight modification of the above indirect argument gives that $\mathscr{V}^{\prime}$ is compact-finite.

Proposition 1.11. If $X$ has Property $Q, X$ is metacompact.

Proof. This is Corollary 1 in [4].

By Remark 1.6, Propositions 1.7, 1.10(a) and 1.11 we have the following:

THEOREM 1.12. For any space $X$, the following implications 
hold: (a) implies (b) implies (c) implies (d) is equivalent to (e) implies $(f)$, where
(a) $X$ is paracompact
(b) $X$ is scc and metacompact
(c) $X$ is mesocompact
(d) $X$ is sequentially mesocompact
(e) $X$ has Property $Q$
(f) $X$ is metacompact.

REMARK 1.13. In view of a result of Michael [11, Th. 2] (a)-(f) are equivalent in collectionwise normal spaces. Examples 1.15 and 1.16 below show that (a) implies (b) and (e) implies (f) cannot be reversed. As yet, the author has no examples showing that (b) implies (c) or (c) implies (d) cannot be reversed.

The following theorem is fundamental and will be used in some of the examples.

THEOREM 1.14. (a) A locally compact space is paracompact if and only if it is mesocompact. (b) A first countable space is paracompact if and only if it is sequentially mesocompact.

Proof. See Theorems 3.7 and 3.10 in [3].

In our examples, $\Omega$ will denote the first uncountable ordinal, and unless otherwise specified, a given set of ordinals will carry the usual order topology and will be called an ordinal space.

EXAMPLE 1.15. A normal mesocompact space which is not collectionwise normal. Michael's subspace [12, Example 2] of Bing's space $F$ [2, Example G]. For the proof that this space has the desired properties see [3, Example 5.1]. A slight modification of this space [4, Th. 18] yields a scc space with Property $Q$ (hence metacompact by Proposition 1.11) which is not collectionwise normal.

EXAMPle 1.16. A metacompact Moore space which is not sequentially mesocompact. R. W. Heath [7, Example 1] gives a metacompact Moore space which is not screenable and so not metrizable. Such a space could not be sequentially mesocompact by [3, Th. 4.2] or our Corollary 1.8.

EXAMPLE 1.17. A normal space which is not mesocompact. The ordinal space $X=\{x ; x<\Omega\}$ is in fact a collectionwise normal locally compact space which is not paracompact. This space can't be meso- 
compact by Theorem 1.14(a). Moreover, $X$ is first countable and so can't be sequentially mesocompact by Theorem 1.14(b).

Definition 1.18. A space $X$ is called an $r$-space [13, p. 985] if each $x \in X$ has a sequence of neighborhoods $\left\{U_{i} ; i \in N\right\}$ such that if $x_{i} \in U_{i}$ for each $i,\left\{x_{i} ; i \in N\right\}$ is contained in a compact subset of $X$.

REMARK 1.19. Clearly, locally compact spaces and first countable spaces are $r$-spaces.

Proposition 1.20. (a) If $X$ is a mesocompact $r$-space, $X$ is paracompact. (b) If $X$ is a scc r-space, $X$ is wce.

Proof. To prove (a), it suffices to show that a compactfinite family $\mathscr{F}$ in $X$ is locally finite. Suppose there exists a point $p$ in $X$ every neighborhood of which meets infinitely many members of $\mathscr{F}$. Let $\left\{U_{i} ; i \in N\right\}$ be a sequence of neighborhoods of $p$ guaranteed by the definition of an $r$-space. We can then find an infinite subcollection $\left\{F_{i} ; i \in N\right\}$ of $\mathscr{F}$ and a sequence of points $\left\{p_{i} ; i \in N\right\}$ such that $p_{i} \in$ $U_{i} \cap F_{i}$ for each $i$. Now $\left\{p_{i} ; i \in N\right\}$ is contained in some compact subset of $X$, which contradicts the compact-finiteness of $\mathscr{F}$.

To prove (b), we modify the proof of Theorem 3 in [4]. Let $\mathscr{U}$ be an open cover of $X$ and $\mathscr{Y}^{-}$a scc open refinement of $\mathscr{U}$. If $V$ is not wcc, there exists an uncountable subcollection $\left\{V_{\alpha} ; \alpha \in A\right\}$ of distinct members of $\mathscr{Y}^{\prime}$ and uncountable point sets $\left\{p_{\alpha} ; \alpha \in A\right\}$ and $q_{\alpha}$; $\alpha \in A\}$ such that $p_{\alpha}, q_{\alpha} \in V_{\alpha}, p_{\alpha} \neq p_{\beta}$ and $p_{\alpha} \neq q_{\beta}$ for $\alpha \neq \beta$. The point set $\left\{p_{\alpha} ; \alpha \in A\right\}$ has a limit point in $X$ but $\left\{q_{\alpha} ; \alpha \in A\right\}$ does not. Since $X$ is an $r$-space, let $\left\{U_{i} ; i \in N\right\}$ be a sequence of neighborhoods of $p$. Then there exists an infinite subset $\left\{p_{i} ; i \in N\right\}$ of $\left\{p_{\alpha} ; \alpha \in A\right\}$ such that $p_{i} \neq p_{j}$ for $i \neq j$ and $p_{i} \in U_{i} \cap V_{i}$. Now $\left\{p_{i} ; i \in N\right\}$ is contained in a compact subset of $X$ and so has a limit point in $X$. Let $q_{i}$ be the member of $\left\{q_{\alpha} ; \alpha \in A\right\}$ corresponding to $p_{i}$. Since $\mathscr{Y}^{-}$is scc, $\left\{q_{i} ; i \in N\right\}$ has a limit point in $X$, contradicting the fact that $\left\{q_{\alpha} ; \alpha \in A\right\}$ does not.

REMARK 1.21. (1) By slightly modifying the proof of (a) in Proposition 1.20 , we can show that a cs-finite family in a sequentially compact space is compact-finite, and so sequential mesocompactness reduces to mesocompactness in sequentially compact spaces. Proposition 1.20(b) slightly generalizes Theorems 3 and 11 in [4] (see Remark 1.19).

(2) A space $X$ is called a $k^{\prime}$-space if whenever $x \in \mathrm{C} 1(A)$, there exists a compact subset $K$ of $X$ such that $x \in \mathrm{C} 1(K \cap A)$ (see [1]). The $k^{\prime}$-spaces include the locally compact spaces and the Frechet spaces. Our interest in $k^{\prime}$-spaces arises from the fact that a regular 
$k^{\prime}$-space is paracompact if and only if it is mesocompact [3, Corollary $3.6]$.

This result, however, does not include Proposition 1.20(a) (see also Example 2.15), since E. Michael has kindly furnished us with an example of an $r$-space which is not a $k^{\prime}$-space:

EXAMPLE 1.22. A separable metric space $X$ and a compact space $Y$ such that $X \times Y$ is not a $k^{\prime}$-space. Let $X$ be an uncountable subset of the reals all of whose compact subsets are countable. Let $Z=X^{\prime} \cup\left\{z_{0}\right\}$ where $X^{\prime}$ is a discrete space of the same cardinality as $X$, and whose neighborhoods of $z_{0}$ in $Z$ are the sets with countable complement in $Z$. Let $Y=\beta Z$. To show that $X \times Y$ is not a $k^{\prime}$ space, pick an $x_{0} \in X$ such that no neighborhood of $x_{0}$ in $X$ is countable and let $h: X \rightarrow Z$ be a one-to-one correspondence with $h\left(x_{0}\right)=z_{0}$. Finally, let $A=\left\{(x, h(x)) \in X \times Y ; x \neq x_{0}\right\}$. Then it can be shown that $\left(x_{0}, z_{0}\right) \in \mathrm{C} 1(A)$ but $\left(x_{0}, z_{0}\right) \notin \mathrm{C} 1(K \cap A)$ for any compact $K \subset X \times Y$. Now $X \times Y$ is an $r$-space since it is not difficult to show that the product of an $r$-space and a locally compact space is an $r$-space.

By Remark 1.6, Propositions 1.10(a) and 1.20 we have:

Theorem 1.23. Let $X$ be a regular $r$-space. Then the following statements are equivalent:

(a) $X$ is paracompact

(b) $X$ is scc and metacompact

(c) $X$ is wcc and metacompact

(d) $X$ is mesocompact.

REMARK 1.24. In [8, Th. 3] it was announced that in a $q$-space ${ }^{1}$ the concepts of paracompactness, $Q_{0}^{*}$ and $Q_{1}^{* 2}$ are equivalent. Since an $r$-space is a $q$-space, we may add (e) $X$ is a $Q_{0}^{*}$-space and $(f) X$ is a $Q_{1}^{*}$-space to Theorem 1.23 .

${ }^{1} X$ is called a $q$-space if every $x \in X$ has a sequence of neighborhoods $\left\{U_{i} ; i \in N\right\}$ such that if $x_{i} \in U_{i}$ for each $i$ and the set $\left\{x_{i} ; i \in N\right\}$ is infinite, then the set $x_{i} ; i \in$ $N\}$ has a limit point in $X$.

${ }^{2}$ A cover $\mathscr{C}$ of $X$. has property $Q_{0}^{*}$ if no indexed point set $\left\{p_{\alpha} ; \alpha \in A\right\}, p_{\alpha} \in U_{\alpha}$, has a limit point where $\mathscr{U}=\left\{U_{\alpha} ; \alpha \in A\right\}^{\cdot}$ A cover $\mathscr{U}=\left\{U_{\alpha} ; \alpha \in A\right\}$ has property $Q_{1}^{*}$ provided for any subcollection $\left\{U_{\alpha} ; \alpha \in A^{*}\right\}$, with $A^{*} \subset A$, if there is an indexed point set $\left\{p_{\alpha} ; \alpha \in A^{*}\right\}, p_{\alpha} \in U_{\alpha}$, having a limit point then there exists a compact set $B$ such that if $\left\{q_{\alpha} ; \alpha \in A^{*}\right\}, q_{\alpha} \in U_{\alpha}$, is any indexed point set then $\left\{q_{\alpha} ; \alpha \in A^{*}\right\}$ has a limit point $p \in B$. A space $X$ is called a $Q_{0}^{*}\left(Q_{1}^{*}\right)$-space if every open cover of $X$ has an open refinement with property $Q_{0}^{*}\left(Q_{1}^{*}\right)$. 
2. Invariance of mesocompactness. In this section, we establish some permanence properties which mesocompact spaces share with paracompact spaces.

Throughout this section, a map will mean a continuous surjection.

Definition 2.1. A closed $\operatorname{map} f: X \rightarrow Y$ is called perfect (or proper) if $f^{-1}(y)$ is compact in $X$ for each $y \in Y$. A set $A$ in $X$ is saturated if $A=f^{-1} f(A)$.

EXAMPLE 2.2. A quotient space of a mesocompact space need not be mesocompact. The ordinal space $X=\{x ; x<Q\}$ is locally compact and locally metrizable and so is the continuous open image of a locally compact metric space [14, Th. 3]. However, we have seen in Example 1.17 that $X$ isn't even sequentially mesocompact.

REMARK 2.3. It is well known that paracompactness is an invariant of a closed map [11]. Less well known, perhaps, is that metacompactness also has this property [15]. We do not know if mesocompactness (or sequential mesocompactness) is an invariant of a closed map. However, for perfect maps we have:

Theorem 2.4. Let $f: X \rightarrow Y$ be a perfect map. Then $X$ is mesocompact (sequentially mesocompact) if and only if $Y$ is mesocompact (sequentially mesocompact).

Proof. Let $X$ be mesocompact and $\mathscr{Y}^{-}=\left\{V_{\alpha} ; \alpha \in A\right\}$ an open cover of $Y$. Then $\left\{f^{-1}\left(V_{\alpha}\right) ; \alpha \in A\right\}$ has a precise compact-finite open $^{3}$ refinement $\mathscr{U}=\left\{U_{\alpha} ; \alpha \in A\right\}$.

For each $y \in Y$, let $G_{y}$ be a finite union of members of $\mathscr{C}$ covering the compact set $f^{-1}(y)$. Since $f^{-1}(y)$ is saturated and $f$ is a closed map, we can find a saturated neighborhood $H_{y}$ of $f^{-1}(y)$ in $X$ such that $H_{y} \subset G_{y}$. For each $y \in Y$, let $V_{\alpha}(y)$ be a member of $\mathscr{y}$ containing $y$. Since $H_{y}$ is saturated and $f$ is closed, $f\left(H_{y}\right)$ is open in $Y$ for each $y$ and so $\mathscr{Y}^{\prime \prime}=\left\{f\left(H_{y}\right) \cap V_{\alpha}(y) ; y \in Y\right\}$ is an open cover of $Y$ which clearly refines $\mathscr{Y}$. Moreover, $\mathscr{V}^{-\prime}$ is compact-finite, for if $K$ is compact in $Y, f^{-1}(K)$ is compact in $X[6$, Th. 1] and so meets at most finitely many members of $\mathscr{C}$ and hence at most finitely many $H_{y}$. Clearly then, $K$ can meet at most finitely many members of $\mathscr{V}^{\prime \prime}$.

Conversely, let $Y$ be mesocompact and $\mathscr{C}=\left\{U_{\alpha} ; \alpha \in A\right\}$ be an open cover of $X$. For each $y \in Y$, let $G_{y}$ be a finite union of members of $\mathscr{U}$ containing the compact set $f^{-1}(y)$. Since $f$ is closed, there exists

${ }^{3}$ A refinement $\mathscr{V}=\left\{V_{\beta} ; \beta \in B\right\}$ of $\mathscr{C}=\left\{U_{\alpha} ; \alpha \in A\right\}$ is called precise if $B=A$ and $V_{\alpha} \subset U_{\alpha}$ for each $\alpha$. If a cover has a compact-finite open refinement, it has a precise compact-finite open refinement (see the proof of Theorem 1.4 in [5, p. 162]). 
a neighborhood $W_{y}$ of $y$ in $Y$ such that $f^{-1}\left(W_{y}\right) \subset G_{y}$ for each $y$. Let $\left\{V_{y} ; y \in Y\right\}$ be a precise compact-finite open refinement of $\left\{W_{y} ; y \in Y\right\}$. Then $\mathscr{U}^{\prime}=\left\{f^{-1}\left(V_{y}\right) \cap U_{\alpha} ; U_{\alpha} \subset G_{y}, y \in Y\right\}$ is a compact-finite open refinement of $\mathscr{U}$.

Replacing compact-finite by cs-finite in the above argument, gives the proof in the sequential mesocompact case, and the theorem is proved.

Since projections parallel to compact factors are perfect maps, the following corollary is immediate.

COROLlaRY 2.5. If $X$ is mesocompact (sequentially mesocompact) and $Y$ is compact, then $X \times Y$ is mesocompact (sequentially mesocompact).

EXAMPLE 2.6. The product of paracompact spaces need not be sequentially mesocompact. Let $X$ be the reals with the upper limit topology [5, p. 66]. Then $X$ is a first countable paracompact space such that $X \times X$ is not normal. Clearly, $X \times X$ can't be sequentially mesocompact by Theorem 1.14(b).

EXAMPLE 2.7. The product of a compact space and a locally compact space need not be mesocompact. Consider the ordinal spaces $X=\{x ; x \leqq \Omega\}$ and $Y=\{y ; y \angle \Omega\}$. Then $X \times Y$ is locally compact and not normal and so can't be mesocompact by Theorem 1.14(a) or Theorem 1.23.

COROLlary 2.8. Let $X$ be mesocompact and $Y$ locally compact and mesocompact. Then $X \times Y$ is mesocompact.

Proof. By hypothesis, $Y$ has a compact-finite (in fact a star finite) open cover $\mathscr{Y}^{-}=\left\{V_{\alpha} ; \alpha \in A\right\}$ such that $\mathrm{C} 1\left(V_{\alpha}\right)$ is compact for each $\alpha \in A$. Let $\mathscr{U}$ be an open cover of $X \times Y$. Then for each $\alpha \in$ $A, X \times \mathrm{C} 1\left(V_{\alpha}\right)$ is mesocompact by Corollary 2.5 and so we can find a (relatively) open cover $\mathscr{U}_{\alpha}$ of $X \times \mathrm{C} 1\left(V_{\alpha}\right)$ which refines $\mathscr{C}_{6}$ and is compact-finite in $X \times \mathrm{C} 1\left(V_{\alpha}\right)$ (and hence in $X \times Y$ ). Let

$$
\mathscr{W}_{\alpha}=\left\{\left(X \times V_{\alpha}\right) \cap U ; U \in \mathscr{U}_{\alpha}\right\}
$$

and let $\mathscr{W}=\left\{\mathscr{W}_{\alpha} ; \alpha \in A\right\}$. Since $\left\{X \times V_{\alpha} ; \alpha \in A\right\}$ is also compactfinite, $\mathscr{W}$ is a compact-finite open cover of $X \times Y$ which refines $\mathscr{U}$.

${ }^{4}$ A map $f: X \rightarrow Y$ is called compact if $f^{-1}(K)$ is compact in $X$ whenever $K$ is compact in $Y$. 
REMARK 2.9. Examples 2.6 and 2.7 show that neither hypothesis for $Y$ in Corollary 2.8 can be dropped.

For compact maps ${ }^{4}$, we have the following result.

Proposition 2.10. Let $f: X \rightarrow Y$ be a compact map and $Y$ a k'space (see Remark 1.21(2)). The $X$ is mesocompact if and only if $Y$ is mesocompact.

Proof. In view of Theorem 2.4, we need only show that $f$ is a closed map. Suppose $f(A)$ is not closed in $Y$ for some closed $A$ in $X$. Then there exists a $y \in \mathrm{C} 1(f(A))-f(A)$. There exists a compact subset $K$ of $Y$ such that $y \in \mathrm{C} 1(K \cap f(A))$. Then $f^{-1}(K) \cap A$ is compact in $X$ since $A$ is closed and $f$ is a compact map. Applying $f$, we have that $K \cap f(A)$ is compact. But then there exist disjoint open sets containing $y$ and $K \cap f(A)$ respectively, a contradiction since $y \epsilon$ $\mathrm{C} 1(K \cap f(A))$.

The following proposition will be used in Example 2.15.

Proposition 2.11. If $f: X \rightarrow Y$ is a closed map and no countably infinite subset of $X$ has a limit point in $X$, then no countably infinite subset of $Y$ has a limit point in $Y$.

Proof. Suppose $\left\{y_{i} ; i \in N\right\}$ is a countably infinite subset of $Y$ with limit point $y$. Pick an $x_{i} \in f^{-1}\left(y_{i}\right)$ for each $i \in N$. The proposition will be proved if we can show that $\left\{x_{i} ; i \in N\right\}$ has a limit point in $f^{-1}(y)$. If not, each $x \in f^{-1}(y)$ has a neighborhood $G_{x}$ which misses $\left\{x_{i} ; i \in N\right\}-\{x\}$. Then $f^{-1}(y) \subset G=\cup\left\{G_{x} ; x \in f^{-1}(y)\right\}$ and so, since $f$ is closed, there exists a neighborhood $V$ of $y$ in $Y$ such that $f^{-1}(V)$ $\subset G$. There exists a $y_{i} \in V$ such that $y_{i} \neq y$, and so $x_{i} \in G$ and $x_{i} \notin f^{-1}(y)$. This is a contradiction which completes the proof.

REMARK 2.12. A standard type argument shows that a closed subspace of a mesocompact space is mesocompact. In view of Proposition 1.7, this also holds for sequentially mesocompact spaces [4, Th. 25]. Arbitrary subspaces of even compact spaces may not be sequentially mesocompact as the subspace $X^{\prime}=\{x ; x<\Omega\}$ of the ordinal space $X=\{x ; x \leqq \Omega\}$ shows (see Example 1.17).

REMARK 2.13. In [10] we asked whether a mesocompact space need be normal or regular. The final two examples answer this question in the negative. 
EXAMPLE 2.14. A mesocompact regular space which is not normal. Briggs [4, Th. 16] gives a scc and metacompact (hence mesocompact by Proposition $1.10(\mathrm{~b})$ ) regular space which is not normal. Let $X=$ $\{x ; x \leqq \Omega\}$, with the discrete topology except at $\Omega$ where it has the order topology. Let $\Omega^{\prime}$ denote the first ordinal such that if $Y=\{y$; $\left.y \leqq \Omega^{\prime}\right\}$, the cardinality of $Y$ is greater than that of $X$. Let $Y$ have the discrete topology except at $\Omega^{\prime}$ where it has the order topology. Let $Z=X \times Y-\left\{\left(\Omega, \Omega^{\prime}\right)\right\}$ with the product topology. We record here that $Z$ is scc since no countable subset of $Z$ has a limit point.

EXAMPLE 2.15. A mesocompact space which is not regular. Let $Z$ be as in Example 2.14. Since $Z$ is not normal, there are disjoint closed subsets $A$ and $B$ of $Z$ such that each neighborhood of $A$ meets each neighborhood of $B$. Indentify say $A$ to a point, and denote the resulting quotient space by $Z / A$. Then $Z / A$ is Hausdorff but not regular $[9$, p. 132G]. The quotient map $f: Z \rightarrow Z / A$ is closed, and so by Remark 2.3 and Proposition 2.11, $Z / A$ is metacompact and sce. Again by Proposition 1.10(b), $Z / A$ is mesocompact.

Finally, the author would like to thank Professor R. C. Briggs, III for making his dissertation available to him, and for some very helpful exchanges.

\section{REFERENCES}

1. A. Arhangelskii, Bicompact sets and the topology of spaces, Soviet Math. Dokl. 4 (1963), 561-564.

2. R. H. Bing, Metrization of topological spaces, Canad. J. Math. 3 (1951), 175-186.

3. J. R. Boone, Some characterizations of paracompactness in k-spaces (to appear).

4. R. C. Briggs, III, A comparison of covering properties in $T_{3}$ and $T_{4}$ spaces, Doctoral Dissertation, Univ. of Houston, 1968.

5. J. Dugundji, Topology, Allyn and Bacon Inc., Boston, 1966.

6. E. Halfar, Compact mappings, Proc. Amer. Math. Soc. 8 (1957), 828-830.

7. R. W. Heath, Screenability, pointwise paracompactness, and metrization of Moore spaces, Canad. J. Math. 16 (1964), 763-770.

8. S. B. Higgins, Some generalizations of paracompactness, Notices Amer. Math. Soc. 16 (1969), 305.

9. J. L. Kelley, General topology, Van Nostrand, Princeton, 1955.

10. V. J. Mancuso, Some properties of mesocompact spaces, Notices Amer. Math. Soc. 16 (1969), 527.

11. E. Michael, Another note on paracompact spaces, Proc. Amer. Math. Soc. 8 (1957), 822-828.

12. - Point-finite and locally finite coverings, Canad. J. Math. 7 (1955), 275279 .

13. - $\boldsymbol{\aleph}_{0}$-spaces, J. Math. Mech. 15 (1966), 983-1002.

14. K. Morita, On decomposition spaces of locally compact spaces, Proc. Japan Acad. 32 (1956), 544-548.

15. J. M. Worrell, Jr., The closed continuous images of metacompact spaces, Port. Math. 25 (1966), 175-179. 
16. J. N. Younglove, Concerning dense metric subspaces of certain non-metric spaces, Fund. Math. 48 (1960), 15-25.

Received August 18, 1969. Presented to the Society April 4, 1969, under the title: Some properties of mesocompact spaces.

ST. JOHN'S UNIVERSITY 



\section{PACIFIC JOURNAL OF MATHEMATICS}

\section{EDITORS}

H. SAMelson

Stanford University

Stanford, California 94305

\section{Richard PIERCE}

University of Washington

Seattle, Washington 98105
J. DugundjI

Department of Mathematics

University of Southern California

Los Angeles, California 90007

BASIL GoRDON*

University of California

Los Angeles, California 90024

\section{ASSOCIATE EDITORS}
E. F. BECKENBACH
B. H. NeumanN
F. WOLE
K. YosHIDA

\section{SUPPORTING INSTITUTIONS}

UNIVERSITY OF BRITISH COLUMBIA

CALIFORNIA INSTITUTE OF TECHNOLOGY

UNIVERSITY OF CALIFORNIA

MONTANA STATE UNIVERSITY

UNIVERSITY OF NEVADA

NEW MEXICO STATE UNIVERSITY

OREGON STATE UNIVERSITY

UNIVERSITY OF OREGON

OSAKA UNIVERSITY

UNIVERSITY OF SOUTHERN CALIFORNIA

\author{
STANFORD UNIVERSITY \\ UNIVERSITY OF TOKYO \\ UNIVERSITY OF UTAH \\ WASHINGTON STATE UNIVERSITY \\ UNIVERSITY OF WASHINGTON \\ * * * * \\ AMERICAN MATHEMATICAL SOCIETY \\ CHEVRON RESEARCH CORPORATION \\ TRW SYSTEMS \\ NAVAL WEAPONS CENTER
}

The Supporting Institutions listed above contribute to the cost of publication of this Journal, but they are not owners or publishers and have no responsibility for its content or policies.

Mathematical papers intended for publication in the Pacific Journal of Mathematics should be in typed form or offset-reproduced, (not dittoed), double spaced with large margins. Underline Greek letters in red, German in green, and script in blue. The first paragraph or two must be capable of being used separately as a synopsis of the entire paper. The editorial "we" must not be used in the synopsis, and items of the bibliography should not be cited there unless absolutely necessary, in which case they must be identified by author and Journal, rather than by item number. Manuscripts, in duplicate if possible, may be sent to any one of the four editors. Please classify according to the scheme of Math. Rev. 36, 1539-1546. All other communications to the editors should be addressed to the managing editor, Richard Arens, University of California, Los Angeles, California, 90024.

50 reprints are provided free for each article; additional copies may be obtained at cost in multiples of 50 .

The Pacific Journal of Mathematics is published monthly. Effective with Volume 16 the price per volume (3 numbers) is $\$ 8.00$; single issues, $\$ 3.00$. Special price for current issues to individual faculty members of supporting institutions and to individual members of the American Mathematical Society: $\$ 4.00$ per volume; single issues $\$ 1.50$. Back numbers are available.

Subscriptions, orders for back numbers, and changes of address should be sent to Pacific Journal of Mathematics, 103 Highland Boulevard, Berkeley, California, 94708.

PUBLISHED BY PACIFIC JOURNAL OF MATHEMATICS, A NON-PROFIT CORPORATION

Printed at Kokusai Bunken Insatsusha (International Academic Printing Co., Ltd.), 7-17, Fujimi 2-chome, Chiyoda-ku, Tokyo, Japan.

* Acting Managing Editor. 


\section{Pacific Journal of Mathematics}

\section{Vol. 33, No. $2 \quad$ April, 1970}

Raymond Balbes and Alfred Horn, Projective distributive lattices ....... 273

John Findley Berglund, On extending almost periodic functions ......... 281

Günter Krause, Admissible modules and a characterization of reduced left

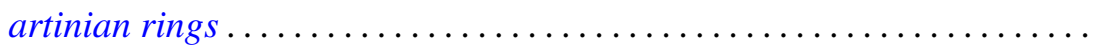

Edward Milton Landesman and Alan Cecil Lazer, Linear eigenvalues and a nonlinear boundary value problem ....................... 311

Anthony To-Ming Lau, Extremely amenable algebras ............... 329

Aldo Joram Lazar, Sections and subsets of simplexes .............. 337

Vincent Mancuso, Mesocompactness and related properties ............. 345

Edwin Leroy Marsden, Jr., The commutator and solvability in a generalized orthomodular lattice .................................. 357

Shozo Matsuura, Bergman kernel functions and the three types of canonical domains.......................................... 363

S. Mukhoti, Theorems on Cesàro summability of series .............. 385

Ngô Van Quê, Classes de Chern et théorème de Gauss-Bonnet ........... 393

Ralph Tyrrell Rockafellar, Generalized Hamiltonian equations for convex problems of Lagrange ................................ 411

Ken iti Sato, On dispersive operators in Banach lattices ............. 429

Charles Andrew Swanson, Comparison theorems for elliptic differential

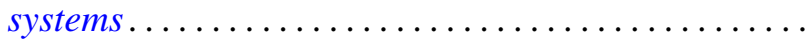

John Griggs Thompson, Nonsolvable finite groups all of whose local subgroups are solvable. II

David J. Winter, Cartan subalgebras of a Lie algebra and its ideals ... 